\title{
Compositional and Structural Evolution during Ball Milling of Ti-based Metallic Glass Powder
}

\author{
Yanuar Rohmat Aji Pradana ${ }^{1}$ \\ ${ }^{1}$ Department of Mechanical Engineering, State University of Malang, Malang City, East Java, Indonesia \\ yanuar.rohmat.ft@um.ac.id
}

\begin{abstract}
Ti42Zr40Ta3Si7.5Sn7.5metallic glass powder prepared for subsequent consolidation by hot pressing were produced by high energy ball milling from amorphous ribbon and both of the compositional and structural evolution on milled powders were studied using XRD and DSC analyses. By using WC vial and balls, the abrasion of milling media occurred causing WC contamination on $<105 \mu \mathrm{m}$ milled powder after 2 cycles of milling. Based on DSC analysis, the contamination clearly reduced the $\Delta$ Txof the alloy up to $48 \%$. On the other hand, the contamination were overcome after milling using SS vial and balls, proved by no crystal peak observed on XRD pattern of all milling cycle levels. However, the thermal stability was noticed to be decreased implying the presence of nanocrystals on the amorphous powder after ball milling and the nanocrystal amount tend to be higher when more milling cycles were applied.
\end{abstract}

Copyright (C) 2017Journal of Mechanical Engineering Science and Technology

All rights reserved

Keywords: Ti-based MG powder, Ball milling, Contamination, Structural evolution

\section{Introduction}

The study of metallic glasses is recently important both in fundamental and application due to their unique and superior properties compared with the crystalline counterparts, which are high strength, high hardness, good corrosion and wear resistance, and excellent soft magnetic property[1]. Titanium-based metallic glass (Ti-based MG) is one of potential candidate materials applied in biomedical field due to its better corrosion and wear resistance, lower Young's modulus, and higher elastic strain limit modulus compared with $\mathrm{Ti}$ and its alloys which are widely used as biomedical implant materials as their good implant requirement properties such as low densityand good biocompatibility[2], [3]. These properties of Ti-based MG can be designed to solve large boneimplant Young's modulus mismatch resulting stress-shielding effects which can damage the surrounding tissue [2], [4], [5].

Although Ti-based MGs become the most attractive candidate of biomaterials among all metallic glasses, $\mathrm{Be}, \mathrm{Ni}$, and $\mathrm{Cu}$ element need to be involved to improve their high GFA, therefore, they can be fabricated into bulk form. But, all of these elements are classified as toxic elements which harmful for human body after long period implantation[6].Ti-Zr-Si-Sn system of metallic glass had been successfully developed by Jang's group. However, this composition possess relatively low GFA due to the absence of toxic elements, so, amorphous structure could be only formed into \pm 18 $25 \mu \mathrm{m}$ thick ribbon. One of the possible ways to obtain bulk and applicableTi-basedmetallic glass is consolidation of its powder constituent by hot pressing process. Usually, this process is isothermally conducted at supercooled liquid region for certain time depends on alloy incubation time for recrystallization.

Ball milling is a simple and flexible processing method to synthesize of metastable material such as amorphous, nanocrystals and solid solution [7], [8]. The main evolution of microstructure during milling generally initiateswith plastic deformation, followed by fracture and subsequent particle welding. Milling parameters to convert ribbon into powder plays an important role to determine structure and properties of generated amorphous powder. The process feasibility and bulk product quality such as mechanical and chemical properties will be influenced by state of initial powder [8], [9].Exceeds milling time and cycles can be the purpose of both powder contamination and crystallization due to the stress and temperature elevation during milling process. On the other hand, the interaction between powder and milling media (both of vial and balls) material also determines the powder composition obtained from milling process. It should be noted the SPEX mill could 
possibly introduce contamination from the grinding media into the milled powders due to its high energetic efficiency.This can obstruct amorphous interface bonding between powders when powder consolidation by hot pressing, then, reducing the mechanical properties of hot pressed sample. In addition, low GFA Ti-based MGs indicating low thermal and mechanical stabilization are susceptible to loss their amorphous nature as a result of thermal and mechanical working during powder milling. So, optimum milling condition and milling media selection must be reached to obtain high quality powder [9], [10]. Nevertheless, the informations regarding milling parameters effect on Ti-based MG powder structure and thermal properties have never been revealed by previous studies.

In this study, the structure and thermal properties of non-toxic Ti-based MGs powder obtained from high energy ball milling using different milling media and number of cycles were investigated due to the contamination and crystallization may occur during milling to provide an overview relating optimum condition and milling parameter selectionwhich can be applied for advance Tibased MGs processing. Ti42Zr40Ta3Si7.5Sn7.5MGwas selected as sample composition due to the absence of toxic elements andits highest GFA among all configuration of the alloy system. The structural and thermal evolution of as spun and powder MG after high energy ball milling were intensively discussed.

\section{Materials and method.}

Alloy ingotswith composition of Ti42Zr40Ta3Si7.5Sn7.5 (at.\%) were firstly prepared by arc melting of representative elements having purity of $99.9 \%$ inside Ti-gettered argon atmosphere. The process is repeated for 4 times by reversing and re-melting the ingot surface in each process to ensure their homogeneity. After the ingots had successfully evacuated from the chamber, they were re-melted and cast to be $\pm 18-25 \mu \mathrm{m}$ MG ribbon by melt spinning method at temperature of $0 \mathrm{oC}$. Subsequently, amorphous ribbon were cut into a small size and put together with ball mill inside the milling vial under argon atmosphere to avoid oxidation during milling process. Tungsten carbide (WC) and stainless steel (SS) ball and vial were utilized during powder milling using 8000M SPEX Sample Preparation Machine.Ball-to-powder ratio (BPR) of 10:1 was used to grind $10 \mathrm{~g}$ fine cut ribbon for each milling process. Due to the low stability of Ti-based MG, the milling procedure was set to grind for $5 \mathrm{~min}$ (very short milling time) followed by a break for 20 min until desired cycle was reached in order to hinder early crystallization. Afterwards, the powder was evacuated from the vial and sieved to obtain only powder having particle size of $<105 \mu \mathrm{m}$. This was chosen to be prepared as subsequent analytical sample.

Structural analysis was conducted on as spun ribbon and milled powder by using Bruker D8A Xray Diffraction (XRD) with scanning rate of 4.8610 min-1. Meanwhile, the thermal properties of them were investigated by Mettler Toledo Differential Scanning Calorimetry (DSC) Instrument to obtain specific temperature (glass transition temperature, $\mathrm{Tg}$, and crystallization temperature, $\mathrm{Tx}$ ). Supercooled liquid (SCL) region $(\triangle \mathrm{Tx}=\mathrm{Tx}-\mathrm{Tg}$ ) obtained from the DSC analysis was defined to be the thermal stability of alloys.

\section{Results and discussion.}

The alloy ingot of Ti42Zr40Ta3Si7.5Sn7.5was successfully fabricated by arc melting and the crystal structure of combined compositions is confirmed by XRD shown on Figure 1(a). Due to the lower GFA of Ti-based BMG compared with other based BMGs, the amorphous structure could only achieved at limited size, therefore, the ingot was cast by using melt spinning to produce ribbon. The XRD pattern of $\pm 18-25 \mu \mathrm{m}$ as spun ribbon is shown on Figure 1(b)exhibiting a broad diffraction peak of $2 \theta$ at $30-50 \mathrm{o}$ without any crystalline Bragg peak indicating the structure is fully amorphous. 

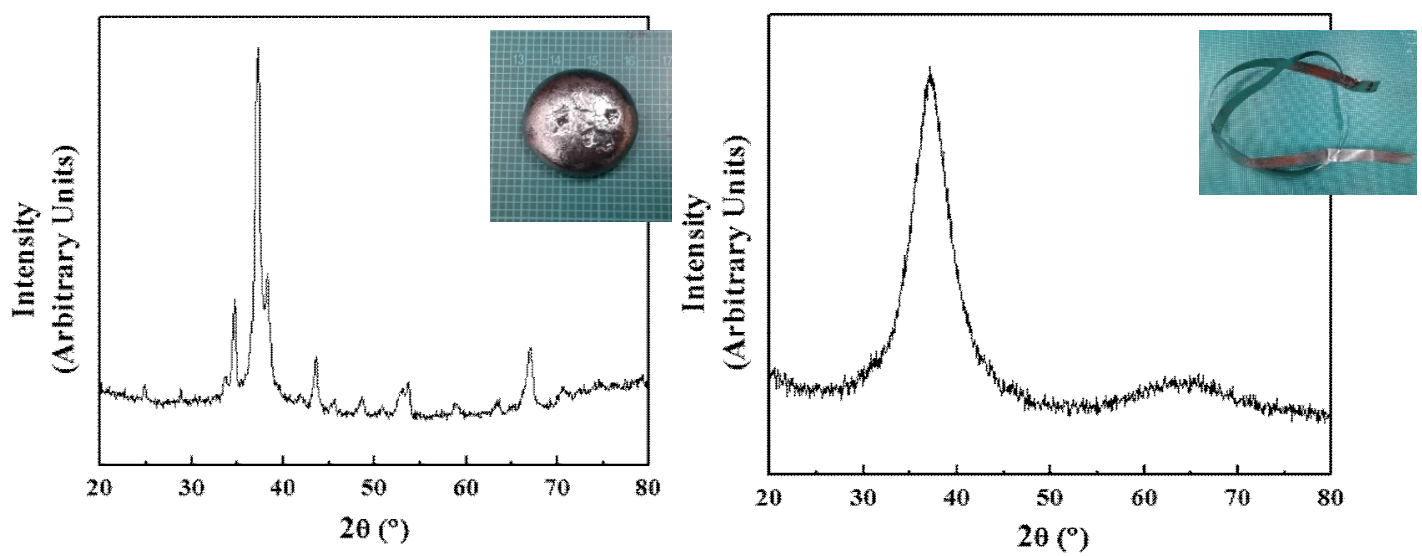

Fig. 1.XRD patterns of $\mathrm{Ti}_{42} \mathrm{Zr}_{40} \mathrm{Ta}_{3} \mathrm{Si}_{7.5} \mathrm{Sn}_{7.5}$ (a) ingot and (b) ribbon

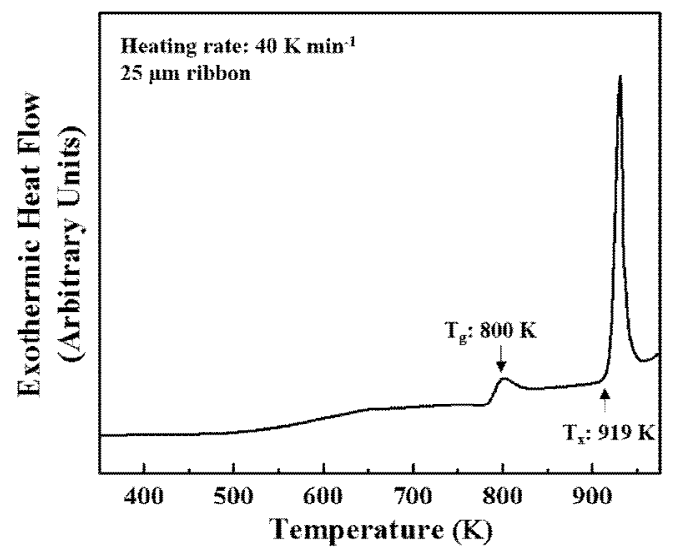

Fig. 2.DSC curve of $\mathrm{Ti}_{42} \mathrm{Zr}_{40} \mathrm{Ta}_{3} \mathrm{Si}_{7.5} \mathrm{Sn}_{7.5} \mathrm{MG}$ ribbon

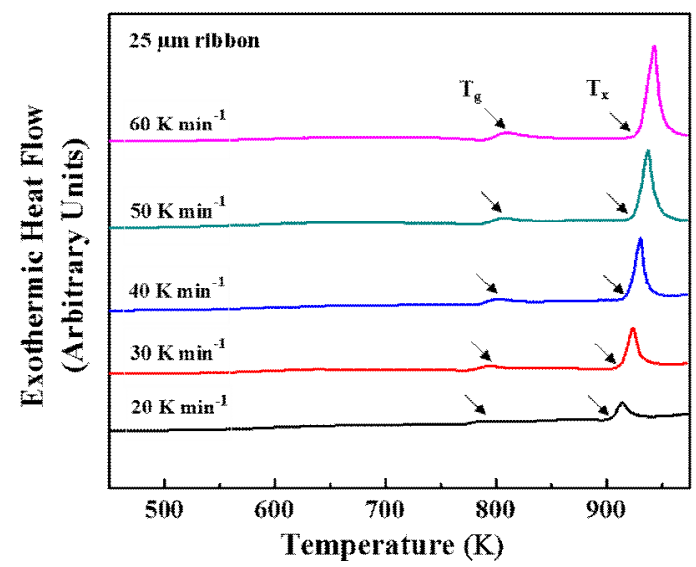

Fig. 3.DSC curves of $\mathrm{Ti}_{42} \mathrm{Zr}_{40} \mathrm{Ta}_{3} \mathrm{Si}_{7.5} \mathrm{Sn}_{7.5}$ ribbon using different heating rates.

To investigate the thermal properties of the alloy, DSC test ranging from $323-973 \mathrm{~K}$ was utilized and the curve of as spun ribbon is shown at Figure 2. Under the heating rate of $40 \mathrm{~K}$ min-1, both glass transition and crystallization temperatures (Tgand Tx) of Ti-based MGwere determined to be 800 and $919 \mathrm{~K}$, respectively, implying relatively wide SCL region (119 K). $\Delta$ Tx was suggested as the criteria to determine the thermal stability of BMGs. 

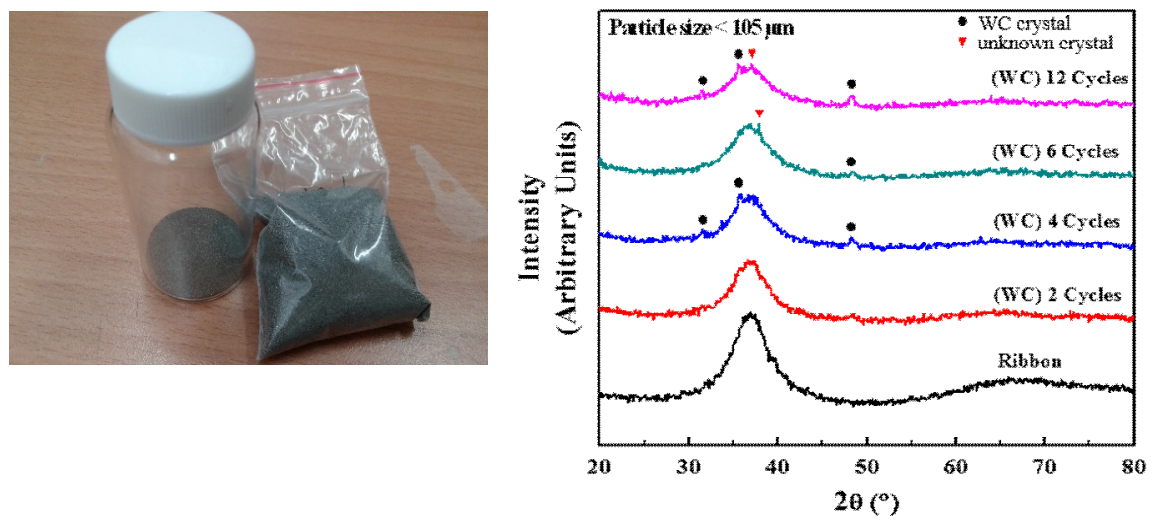

Fig. 4.(a) $\mathrm{Ti}_{42} \mathrm{Zr}_{40} \mathrm{Ta}_{3} \mathrm{Si}_{7.5} \mathrm{Sn}_{7.5} \mathrm{MG}$ powder after ball milling and (b) XRD pattern of powder milled by different milling cycles.
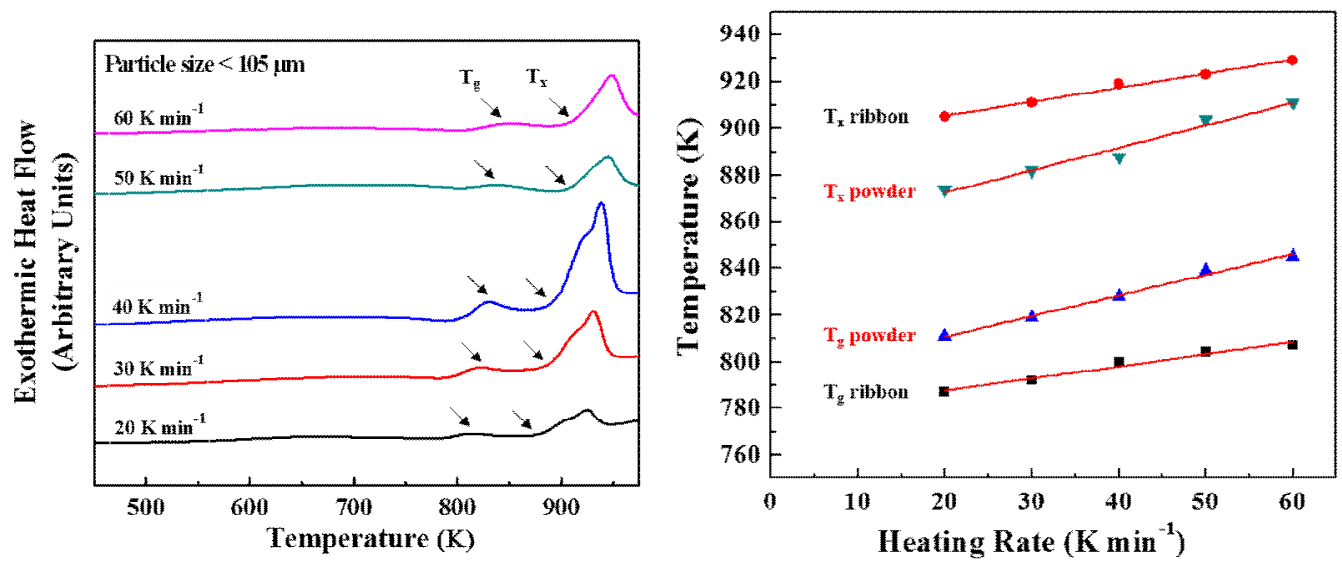

Fig. 5.(a) DSC curves of WC-contaminated MG powder using different heating rates and (b) linearly plot of real Tg and Tx on WC-contaminated MG powder compared with the as-spun ribbon

Due to the isothermal condition is applied during subsequent hot pressing, it is necessary to find both of the real $\mathrm{Tg}$ and Tx by DSC analysis using different heating rates to predict the width SCL regionwhen using heating rate of $0 \mathrm{~K}$ min-1 (isothermal). The results are shown on Figure 3 and the real $\mathrm{Tg}$ and $\mathrm{T} \neg \mathrm{x}$ of ribbon were linearly fit to be 777 and $893 \mathrm{~K}$, thus, $\Delta$ Txwas calculated as $116 \mathrm{~K}$.

Afterwards, high energy ball mill was used to produce the powder from small cut ribbon. Tungsten carbide (WC) vial and ball were firstly utilized to grind the ribbon. Different milling cycles was conducted to vary the powder result. From XRD pattern on Figure 4 (b), no structural change is observed by 2 cycles of milling indicating the structure was still amorphous. By further milling cycles, the crystal peaks dominated by WC crystal are clearly appeared indicating the presence of crystal phase around the milled powders. In addition, the higher amount of crystal phases was generated by 12 cycles of milling with the presence of unknown crystal other than WC.

To investigate the WC contamination on structural and thermal property of milled powders, the DSC analysis of contaminated powder were done using different heating rates. The DSC curves are shown on Figure 5 (a). By using similar method to predict the real specific temperaturesof ribbon, the Tg and Tx of contaminated powders were determined to be 793 and $853 \mathrm{~K}$ resulting $\Delta$ Txof only $60 \mathrm{~K}(48 \%$ of reduction) (Figure 5 (b)). This indicated the degradation of thermal stability of amorphous powder by the presence of certain amount of WC from vial and ball abrasion after high energy ball milling. The higher Tg gives the higher opportunity for alloy to suffer oxidation during heating. In addition, lower Tx generates rapid crystallization when alloys are put in practical usewithin SCL region, i.e. superplastic forming. 
Table 1. Comparison of real glass transition temperature, crystallization temperature, and width of SCL region between WC-contaminated MG powders and as spun ribbon

\begin{tabular}{cccc}
\hline Sample & $\mathbf{T}_{\mathbf{g}}[\mathbf{K}]$ & $\mathbf{T}_{\mathbf{x}}[\mathbf{K}]$ & $\mathbf{\Delta}_{\mathbf{x}}[\mathbf{K}]$ \\
\hline Ribbon & 777 & 893 & 116 \\
\hline Powder & 793 & 853 & 60 \\
\hline
\end{tabular}
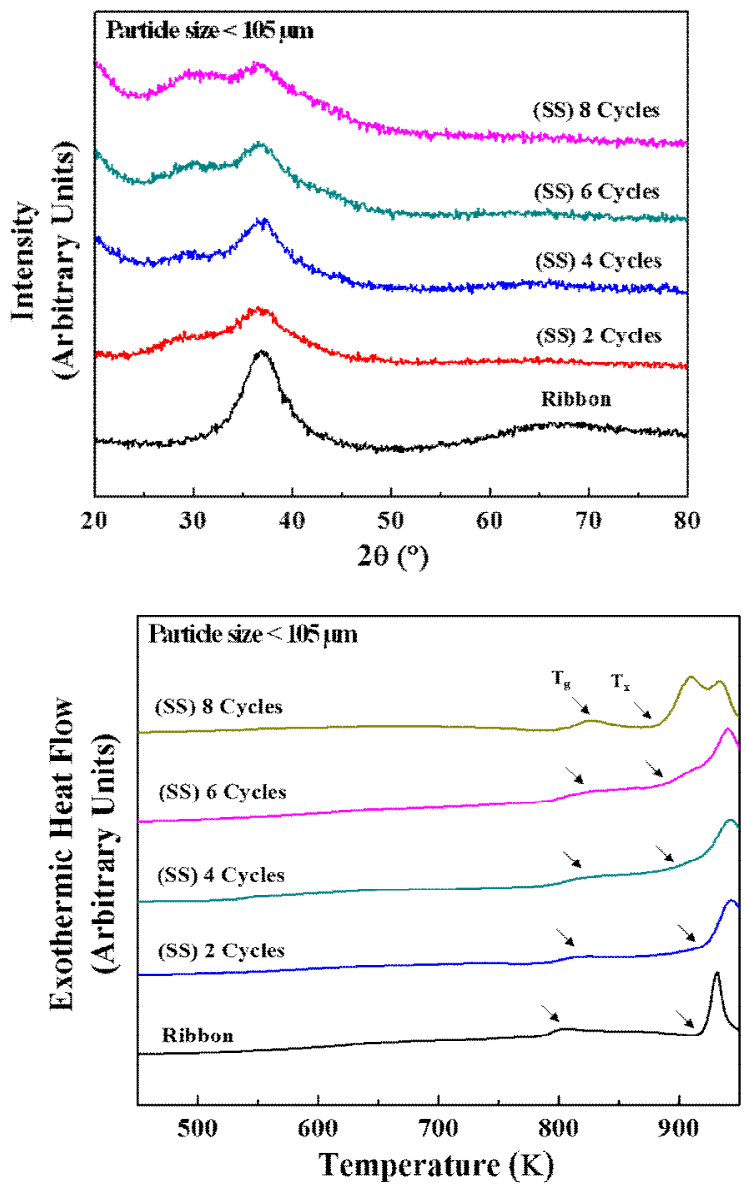

Fig. 6. (a) XRD patterns and (b) DCS curves of milled powder for different milling cycles by using SS vial and balls.

On the other hand, stainless steel (SS) vial and balls were also used to produce the MG powder from small cut ribbons. The XRD pattern of 2, 4, 6, 8 cycles completed with its ribbon are delivered on Figure 6 (a). It is clearly shown that the contamination of milling media on milled powders are hindered by using SS vial and balls proved by the absence of Fe crystal peak. However, weak diffraction crystal peak at around main amorphous hump appeared at powder sample resulted by 4 cycles of milling, indicating the formation crystals with nanometer size(nanocrystals) in the amorphous. By the higher milling cycles, the more peaks are observed to be separated in other region. The limitation of XRD analysis to detect nanocrystals made the clear peaks on the pattern are not achieved, therefore, only weak signal could be performed[11].

Due to relatively imprecise results of XRD to reveal the presence of nanocrystal, the subsequent DSC analysis was conducted to clarify the structural and thermal properties change after ball milling. As described on Figure 6 (b), after ball milling for 2 cycles, bothof Tg and Txare lessvisible 
and the remain crystallization peak is slightly broadened. These phenomena are consistently observed at higher cycles of milling powders, moreover, the one additional crystallization peak rise after 8 cycles of milling. On the other hand, the $\Delta$ Txare gradually decreased with higher milling cycles. It indicates that the thermal stability remain reduced after high energy ball milling. The degradation of amorphous nature by minor crystallization of powder is considered to be occurred after the process, therefore, using high cycle parameter of ball milling remains disadvantage to produce powder for subsequent hot pressing.

The change of alloy composition due to abrasion and contamination of milling media,direct crystallization and oxidation caused by rise of local temperature at collision region on powder trapped by milling balls or milling ball-vial, and stress induced crystallization from shear bands development which act as preferred site for nanocrystals precipitation are the possible reasons ofthe structural evolution on MG powder during ball milling[9], [12].

By using WC vial and balls, the contamination was possibly occurred due to the high strength of Ti based MG powder damaging the WC particle bonding resulting abrasion on milling media wall and finally contaminate the powder. These phenomena are clearly proved by the presence of WC crystal peaks on XRD pattern. By using higher milling cycles, the contamination is observed to reach higher level consistent to the higher intensity of WC peaks. By the fact that the milling was done under Ar atmosphere, the presence of oxygen in milling chamber could be neglected. Increase of oxygen content related with the increase of powder surface-to-volume inducing oxidation when heat rise during milling process. However, this case was hindered due to the powder only milled for short time which is not sufficient to generate heat for oxidation. From the DSC test, the contamination clearly degrade the thermal stability of MG powder, therefore, reduce their properties for subsequent hot pressing.

While SS vial and balls used, the abrasion and contamination of milling media on milled powders are not occurred by examining the XRD result. However the thermal stability change observed by DSC test indicates the structural evolution of milled powder compared with its ribbon counterpart. Similar with the latest one, the oxidation could be neglected due to the presence of only very small amount of oxygen in Ar atmosphere and the absence heat during short time milling process. On the other hand, the deformation of MG at temperature lower than Tg is determined by the formation and movement of shear band which localized in weakest site of MG [13]. The strength of material inside the shear band is reduced during shear band formation, afterwards, deformation takes place along the shear bands [12], [14]. This suggest that during the MG deformation, viscous flow inside the shear bands is improved and structural changes may happen within the shearband.The rapid propagation of shear band induces the local temperature rise on shear band tip playing important role in the formation of nanocrystals on low GFA MG as shown by most of Tibased MG without any toxic element composition[12], [15]. This possibly describes the presence of nanocrystals inside the amorphous powder, which reduce the thermal stability of the MG. By the higher cycles, the amount of nanocrystals are also improved. The presence of optimum amount of nanocrystals inside the amorphous phase may improve its mechanical properties, however, those on MG powder are not beneficial because it can deteriorate the thermal properties and impede the powder-to-powder interface during powder consolidation by hot pressing.

\section{Conclusion.}

Ball milling has been progressively applied to produce Ti42Zr40Ta3Si7.5Sn7.5metallic glass powder from amorphous ribbon using both of WC and SS milling media. From XRD analysis, WC particle contamination were clearly observed after 4 cycles of milling indicating deteriorate effect on MG powder after ball milling using WC vial and balls. The DSC test of contaminated powder revealed the reduction of their thermal stability compared with the ribbon. The contamination and its thermal stability degradation effect remained higher by using more cycles of milling. On the other hand, contamination of milling media was overcome when SS vial and balls were utilized during ball milling. However, the presence of nanocrystals were observed after ball milling as confirmed by XRD and DSC analysis. The amount of nanocrystals tend to be higher when the higher milling cycles were used. These phenomena may degrade the properties of MG powder for subsequent consolidation by hot pressing. 


\section{References}

[1] A. Inoue, "Stabilization of Metallic Supercooled Liquid," Acta Mater., vol. 48, pp. 279-306, 2000.

[2] M. Calin et al., "Designing biocompatible Ti-based metallic glasses for implant applications," Mater. Sci. Eng. C, vol. 33, no. 2, pp. 875-883, 2013.

[3] H. C. Lin et al., "Designing a toxic-element-free Ti-based amorphous alloy with remarkable supercooled liquid region for biomedical application," Intermetallics, vol. 55, pp. 22-27, 2014.

[4] J. J. Oak and A. Inoue, "Attempt to develop Ti-based amorphous alloys for biomaterials," Mater. Sci. Eng. A, vol. 448-451, pp. 220-224, 2007.

[5] J.-J. Oak, D. V. Louzguine-Luzgin, and A. Inoue, "Fabrication of Ni-free Ti-based bulk-metallic glassy alloy having potential for application as biomaterial, and investigation of its mechanical properties, corrosion, and crystallization behavior," J. Mater. Res., vol. 22, no. 5, pp. 1346-1353, 2007.

[6] [6] W. M. Elshahawy, I. Watanabe, and P. Kramer, "In vitro cytotoxicity evaluation of elemental ions released from different prosthodontic materials," Dent. Mater., vol. 25, no. 12, pp. 1551-1555, 2009.

[7] P. Ramasamy, R. N. Shahid, S. Scudino, J. Eckert, and M. Stoica, "Influencing the crystallization of Fe $80 \mathrm{Nb} 10 \mathrm{~B} 10$ metallic glass by ball milling,” J. Alloys Compd., vol. 725, pp. 227-236, 2017.

[8] J. Bednar????k et al., "Crystallization of CoFeSiB metallic glass induced by long-time ball milling," $J$. Non. Cryst. Solids, vol. 337, no. 1, pp. 42-47, 2004.

[9] C. L. Chen and C. L. Huang, "Milling media and alloying effects on synthesis and characteristics of mechanically alloyed ODS heavy tungsten alloys," Int. J. Refract. Met. Hard Mater., vol. 44, pp. 19-26, 2014.

[10] M. Cabeza et al., "Effect of high energy ball milling on the morphology, microstructure and properties of nano-sized TiC particle-reinforced 6005A aluminium alloy matrix composite," Powder Technol., vol. 321, pp. 31-43, 2017.

[11] A. Inoue and C. Suryanarayana, Bulk metallic glasses. 2011.

[12] C. A. Schuh, T. C. Hufnagel, and U. Ramamurty, "Mechanical behavior of amorphous alloys," Acta Mater., vol. 55, no. 12, pp. 4067-4109, 2007.

[13] A. S. Argon, "Plastic Deformation in metallic glasses, "Acta Mater., vol. 27, issue 1, pp. 47-58, 1979.

[14]P. S. Steif, F. Spaepen, and J. W. Hutchinson, "Strain localizationin amorphous metals," Acta Metal, vol. 30 , issue 2, pp. 447-455, 1982 .

[15] J. J. Lewandowski and A. L. Greer, "Temperature rise at shear bands in metallic glasses," Nature Mater, vol. 5, pp. 15-18, 2006. 